\title{
Development of an Algorithm for Automatic Image Detection of Biological Particles in Transmission Electron Microscopy Images
}

\author{
M. C. Proença****, J. F. Moura Nunes*** and A. P. Alves de Matos**,**** \\ * Laboratório de Óptica, Lasers e Sistemas, Faculdade de Ciências da Universidade de Lisboa, Ed. C8, \\ 1749-016 Lisboa \\ ** Centro de Estudos do Ambiente e do Mar, Faculdade de Ciências da Universidade de Lisboa, Ed. \\ C8, 1749-016 Lisboa \\ *** Laboratório de Microscopia Electrónica, Instituto Português de Oncologia, Rua Prof. Lima Basto, \\ 1099-023 Lisboa \\ **** Serviço de Anatomia Patológica, Hospital Curry Cabral, Rua da Beneficência 8, 1069-166 \\ Lisboa
}

Automatic image processing of transmission electron microscopy images (TEM) is a utopia often pursued, considering the thousands of images necessary to ensure a high resolution $3 \mathrm{D}$ reconstruction of virus particles or other macromolecular machines.

The low contrast due to the acquisition process of the samples and the presence of biological debris in the background, along with the artifacts inherent to the negative staining method, difficult the automation task. We were looking for a fully automatic procedure that detects all adenovirus particles, whatever the capsides remains intact or perforated, regardless of the background.

The algorithmic approach implemented relies on the use of an entropy proportion image built by computation of the entropy figure for two concentric structuring elements in a moving window all over the image. This ratio presents localized minima when it coincides with a virus particle, empty or not: it is the virus capsid reinforced by the stain accumulation that provides an annular region of very low entropy, when compared to the entropy of the local footprint (Figure 1).

The credibility of the candidate points as virus particles is verified in a 3D texture image: four profiles at each point provide 8 features reflecting morphological and radiometric characteristics (Figure 2). These features are computed and evaluated according a set of acceptance conditions - which is characteristic of one type of particles and should work for images of the same virus particles acquired in the same conditions, with the necessary adjustments to the particle dimensions targeted.

A final validation is done in the set of accepted candidates (Figure 3): a three dimensional space of entropy-proportion images computed in filtered versions of the original image provides the data frame where the points corresponding to the virus particles do form a cluster and the outliers can be automatically eliminated.

The algorithm was developed in Matlab R2010a, using a mix of proprietary functions and routines specifically developed for this purpose. 

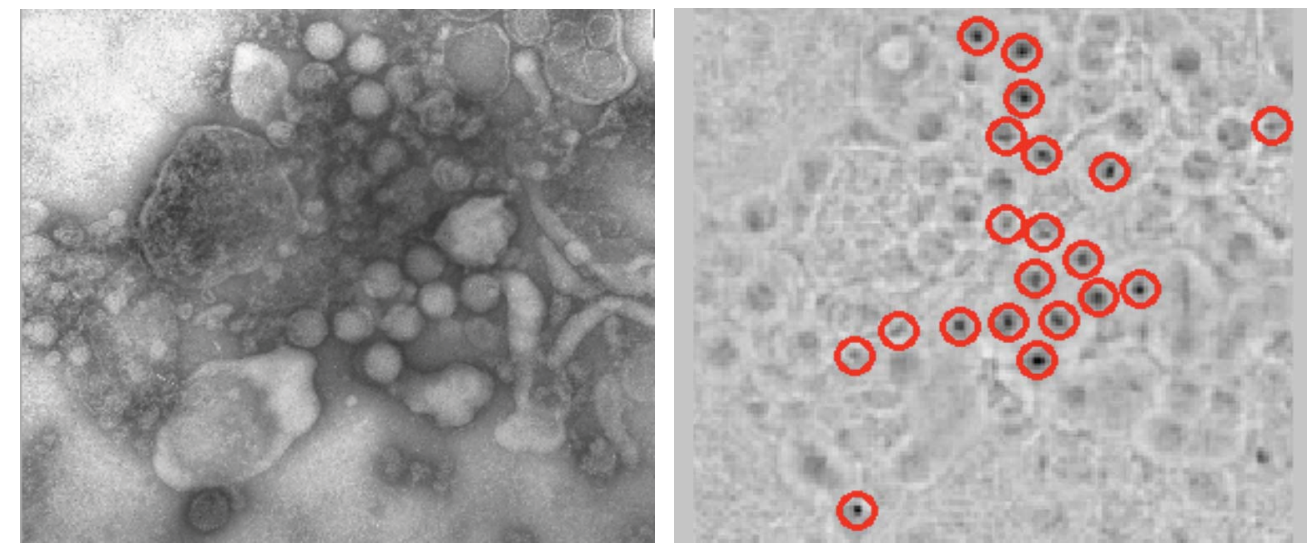

Figure 1. Original image (left) and first image of the local measure of entropy, with minima encircled (right): even perforated and broken capsides can be detected in a confusing background; some adenovirus particles can be identified beyond doubt, but not others.
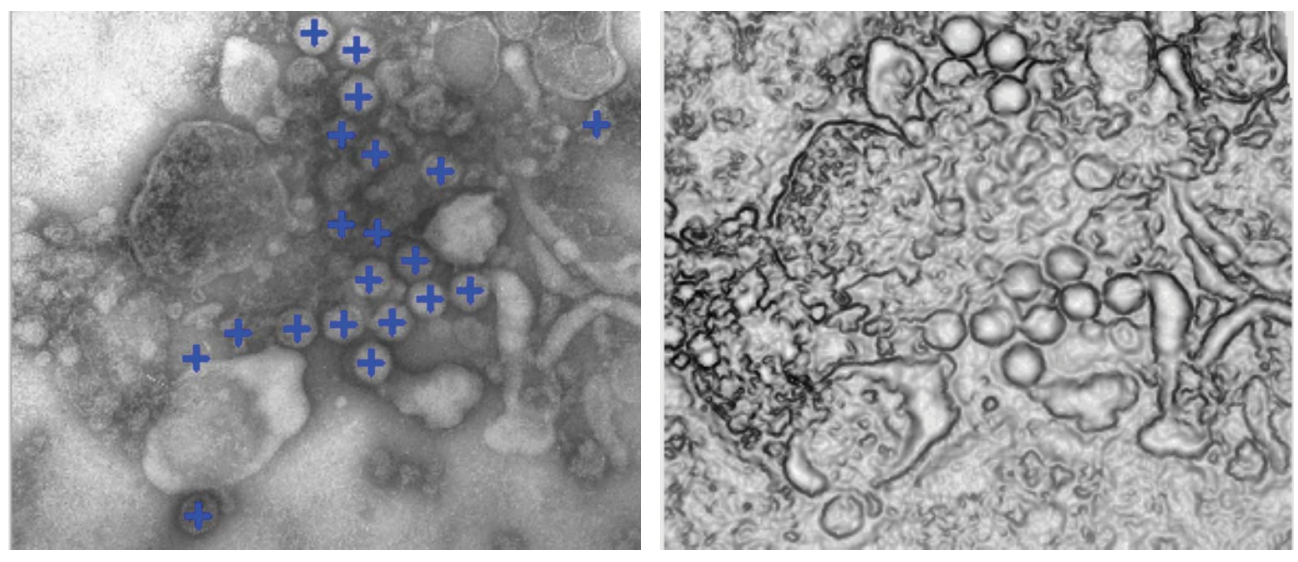

Figure 2. Candidate points (left) and local texture image to evaluate the likelihood of each candidate (right).
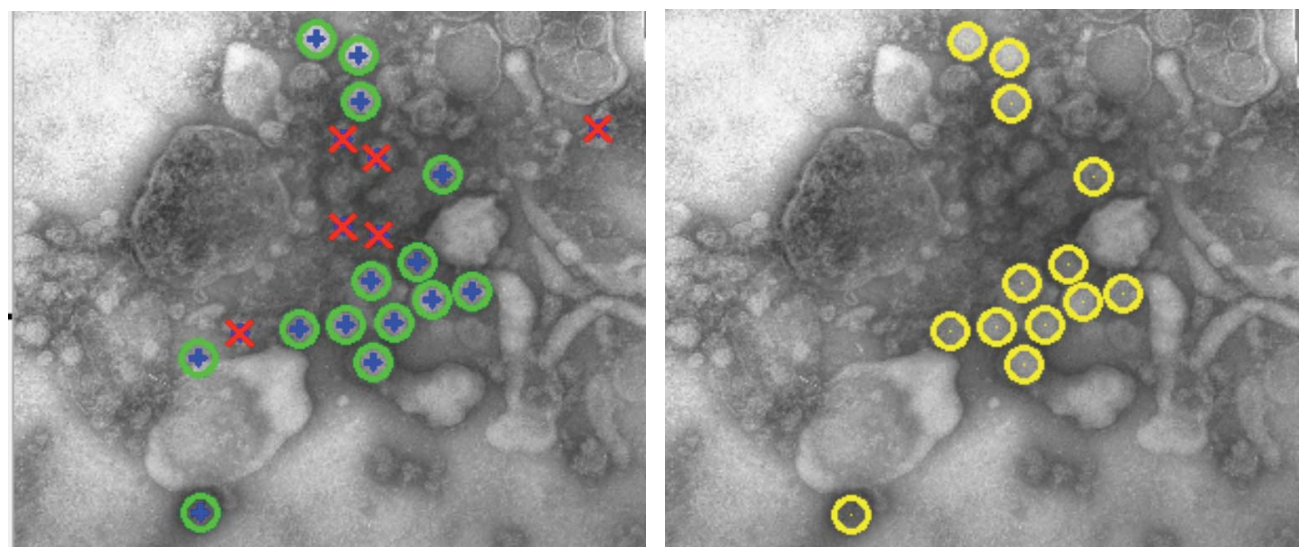

Figure 3. Left: accepted (green circle) and rejected (red cross) candidates using the set of features derived from the local profiles and right: the result of the final validation showing the elimination of the remaining false positive. 